\title{
Intraconfigurational Transition due to Surface-Induced Symmetry Breaking in Noncovalently Bonded Molecules
}

Bouatou, Mehdi; Harsh, Rishav; Joucken, Frédéric; Chacon, Cyril; Repain, Vincent; Bellec, Amandine; Girard, Yann; Rousset, Sylvie; Sporken, Robert; Gao, Fei

Total number of authors:

15

Published in:

Journal of Physical Chemistry Letters

Link to article, DOI:

10.1021/acs.jpclett.0c02407

Publication date:

2020

Document Version

Peer reviewed version

Link back to DTU Orbit

Citation (APA):

Bouatou, M., Harsh, R., Joucken, F., Chacon, C., Repain, V., Bellec, A., Girard, Y., Rousset, S., Sporken, R., Gao, F., Brandbyge, M., Dappe, Y. J., Barreteau, C., Smogunov, A., \& Lagoute, J.' (2020). Intraconfigurational Transition due to Surface-Induced Symmetry Breaking in Noncovalently Bonded Molecules. Journal of Physical Chemistry Letters, 11(21), 9329-9335. https://doi.org/10.1021/acs.jpclett.0c02407

\section{General rights}

Copyright and moral rights for the publications made accessible in the public portal are retained by the authors and/or other copyright owners and it is a condition of accessing publications that users recognise and abide by the legal requirements associated with these rights.

- Users may download and print one copy of any publication from the public portal for the purpose of private study or research.

- You may not further distribute the material or use it for any profit-making activity or commercial gain

- You may freely distribute the URL identifying the publication in the public portal 


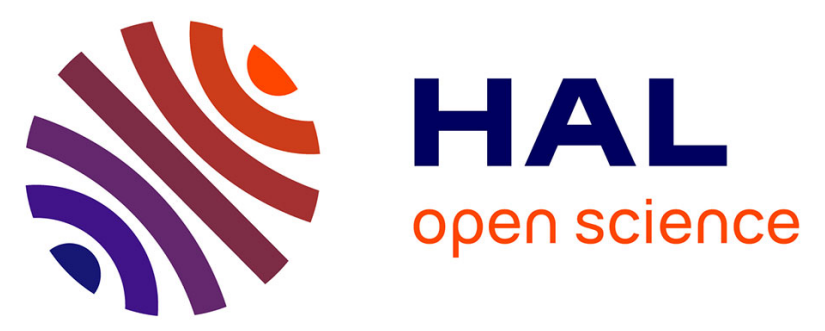

\title{
Intraconfigurational Transition due to Surface-Induced Symmetry Breaking in Non-Covalently Bonded Molecules
}

\author{
Mehdi Bouatou, Rishav Harsh, Frédéric Joucken, Cyril Chacon, Vincent \\ Repain, Amandine Bellec, Yann Girard, Sylvie Rousset, Robert Sporken, Fei \\ Gao, et al.
}

\section{To cite this version:}

Mehdi Bouatou, Rishav Harsh, Frédéric Joucken, Cyril Chacon, Vincent Repain, et al.. Intraconfigurational Transition due to Surface-Induced Symmetry Breaking in Non-Covalently Bonded Molecules. Journal of Physical Chemistry Letters, American Chemical Society, 2020, 11 (21), pp.9329-9335. 10.1021/acs.jpclett.0c02407 . hal-02992071

\section{HAL Id: hal-02992071 \\ https://hal.archives-ouvertes.fr/hal-02992071}

Submitted on 6 Nov 2020

HAL is a multi-disciplinary open access archive for the deposit and dissemination of scientific research documents, whether they are published or not. The documents may come from teaching and research institutions in France or abroad, or from public or private research centers.
L'archive ouverte pluridisciplinaire HAL, est destinée au dépôt et à la diffusion de documents scientifiques de niveau recherche, publiés ou non, émanant des établissements d'enseignement et de recherche français ou étrangers, des laboratoires publics ou privés. 


\section{Intraconfigurational Transition due to}

\section{Surface-Induced Symmetry Breaking in Non-Covalently Bonded Molecules}

Mehdi Bouatou, ${ }^{\dagger}$ Rishav Harsh, ${ }^{\dagger}$ Frédéric Joucken, ${ }^{\ddagger}, \|$ Cyril Chacon ${ }^{\dagger}{ }^{\text {Vincent }}$ Repain, $^{\dagger}$ Amandine Bellec, ${ }^{\dagger}$ Yann Girard ${ }^{\dagger}$ Sylvie Rousset, ${ }^{\dagger}$ Robert Sporken, ${ }^{\ddagger}$ Fei Gao, Mads Brandbyge, Y Yannick J. Dappe, ${ }^{\S}$ Cyrille Barreteau, ${ }^{\S}$ Alexander Smogunov, ${ }^{\S}$ and Jérôme Lagoute ${ }^{*, \dagger}$

†Université de Paris, Laboratoire Matériaux et Phénomènes Quantiques, CNRS, F-75013, Paris, France

$\ddagger$ Research Center in Physics of Matter and Radiation (PMR), Université de Namur, 61 Rue de Bruxelles, 5000 Namur, Belgium

ICenter for Nanostructured Graphene, Department of Physics, Technical University of Denmark, DK-2800 Kongens Lyngby, Denmark

$\S S P E C, C E A, C N R S$, Université Paris-Saclay, CEA Saclay, 91191 Gif-sur-Yvette Cedex, France

||Present address: Department of Physics, University of California, Santa Cruz, California 95060, USA

E-mail: jerome.lagoute@univ-paris-diderot.fr 


\begin{abstract}
The interaction of molecules with surfaces plays a crucial role in the electronic and chemical properties of supported molecules and needs a comprehensive description of interfacial effects. Here, we unveil the effect of the substrate on the electronic configuration of iron porphyrin molecules on $\mathrm{Au}(111)$ and graphene, and we provide a physical picture of the molecule-surface interaction. We show that the frontier orbitals derive from different electronic states depending on the substrate. The origin of this difference comes from molecule-substrate orbital selective coupling caused by reduced symmetry and interaction with the substrate. The weak interaction on graphene keeps a ground state configuration close to the gas phase, while the stronger interaction on gold stabilizes another electronic solution. Our findings reveal the origin of the energy redistribution of molecular states for non-covalently bonded molecules on surfaces.
\end{abstract}

\title{
Graphical TOC Entry
}

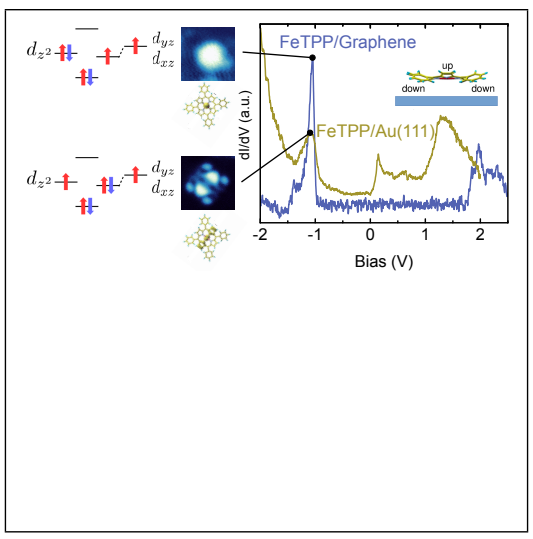


When a molecule is brought in contact with a conducting substrate, its electronic spectrum is renormalized due to electronic coupling, charge transfer and the image charge effect. As a consequence, molecules on surfaces have different properties as compared to their gas phase. ${ }^{1-4}$ These effects typically only change the energy position of the molecular states but not their relative ordering. Consequently, changing the substrate material may preserve the nature of the molecular states involved in the frontier orbitals. ${ }^{5-7}$ Planar molecules such as porphyrin and their derivatives are ideal model systems to investigate the moleculesurface interaction at the atomic level using local probe techniques. ${ }^{8}$ Among these class of chemical compounds, particular attention has been focused on iron porphyrins because of their essential role in biological process involving a heme prosthetic group. ${ }^{9}$ The electronic structure of the free molecules has been thoroughly investigated both experimentally and theoretically revealing the dependence of the spectroscopic state to the environment of the iron atom that gives a ground state either in a low $(\mathrm{S}=0)$, high $(\mathrm{S}=2)$ or intermediate spin state $(\mathrm{S}=1) \cdot{ }^{10}$ From the theoretical side, density functional theory (DFT) indicates that the tetraphenyl iron porphyrin (FeTPP) molecule represents quite a complex multi-configuration case where many intermediate $\mathrm{S}=1$ solutions are possible depending on the filling of Fe dorbitals while $\mathrm{S}=2$ states are generally found much higher in energy. ${ }^{10-13}$ These multiple $\mathrm{S}=1$ solutions are very close in energy and their relative positions depend noticeably on the calculational method and on the type of exchange-correlation functional. ${ }^{13}$ It is generally found that the ground state of isolated FeTPP is an intermediate spin $S=1$ state ${ }^{3} A_{2 g}$ having $\left(d_{x y}\right)^{2}\left(d_{\pi}\right)^{2}\left(d_{z^{2}}\right)^{2}$ electronic configuration. ${ }^{10-13}$ For FeTPP on $\mathrm{Au}(111)$, the ground state remains unclear, since $S=1^{14,15}$ and $S=2^{16}$ have been proposed. This discrepancy in theoretical predictions may be presumably related to different values of Hubbard U parameter on Fe atom which is known to favor generally high spin states. In Refs.[ 14,15], $\mathrm{U}=3$ and 2 $\mathrm{eV}$ were used, respectively, so that intermediate spin state $\mathrm{S}=1$ was found to have the lowest energy. In Ref.[ 16] a larger value of $\mathrm{U}=6 \mathrm{eV}$ was employed which could stabilize high spin $\mathrm{S}=2$ state. With such sensitive molecular species, the nature of the substrate is expected 
to play an important role in the ground state of adsorbed molecules. This can be exploited to open a new route for interfacial engineering, but experimental data and a comprehensive theoretical description are still needed.

Here, we investigate the role of the substrate on the properties of physisorbed FeTPP molecules. We combine low temperature scanning tunneling microscopy (STM) and DFT calculations to compare the properties of FeTPP on $\mathrm{Au}(111)$ and on graphene. We show that, although the molecules are physisorbed in all cases, the substrate does not simply shift and broaden the molecular states but also redistributes the energy ordering of Ferelated molecular states. This effect is triggered by different hybridization strength of Fe orbitals with the substrate due to their different spatial localization on the molecule. An important consequence of this interaction is that the frontier orbitals have different nature and distribution and involve different electronic states (with different filling of Fe $d$ states) depending on the substrate.

On $\mathrm{Au}(111)$ the FeTPP molecules are organized in small clusters and single molecules (Figure 1a). Some molecules exhibit a bright spot in the center corresponding to a chlorine bonded to a FeTPP and forming a FeTPP-Cl species. ${ }^{14}$ In the following we concentrate on FeTPP only, since the spectroscopic investigation of FeTPP-Cl often removes the $\mathrm{Cl}$ ion leading to tip contamination. On pristine graphene, in contrast to $\mathrm{Au}(111)$, single molecules were not stable enough to be observed by STM, only islands of self-assembled molecules were observed after room temperature annealing of the sample (Figure 1b). This is indicative of a weaker molecule-substrate interaction as compared to gold. In these islands, FeTPP-Cl and FeTPP can be easily distinguished.

The spectroscopy of FeTPP on $\mathrm{Au}(111)$ measured on a pyrrole group is shown in Figure 1c. Three peaks appear at $-1.1 \mathrm{~V}, 0.15 \mathrm{~V}$ and $1.3 \mathrm{~V}$, which are localized on two pyrrole groups (peaks at $-1.1 \mathrm{~V}$ and $0.15 \mathrm{~V}$ ) or between the pyrroles (peak at $1.3 \mathrm{~V}$ ), as shown in Figure 1d-f. These results agree with previously reported STM measurements, however, contrary to the previous interpretation, ${ }^{16}$ we attribute the origin of the peaks to a HOMO and a split LUMO 

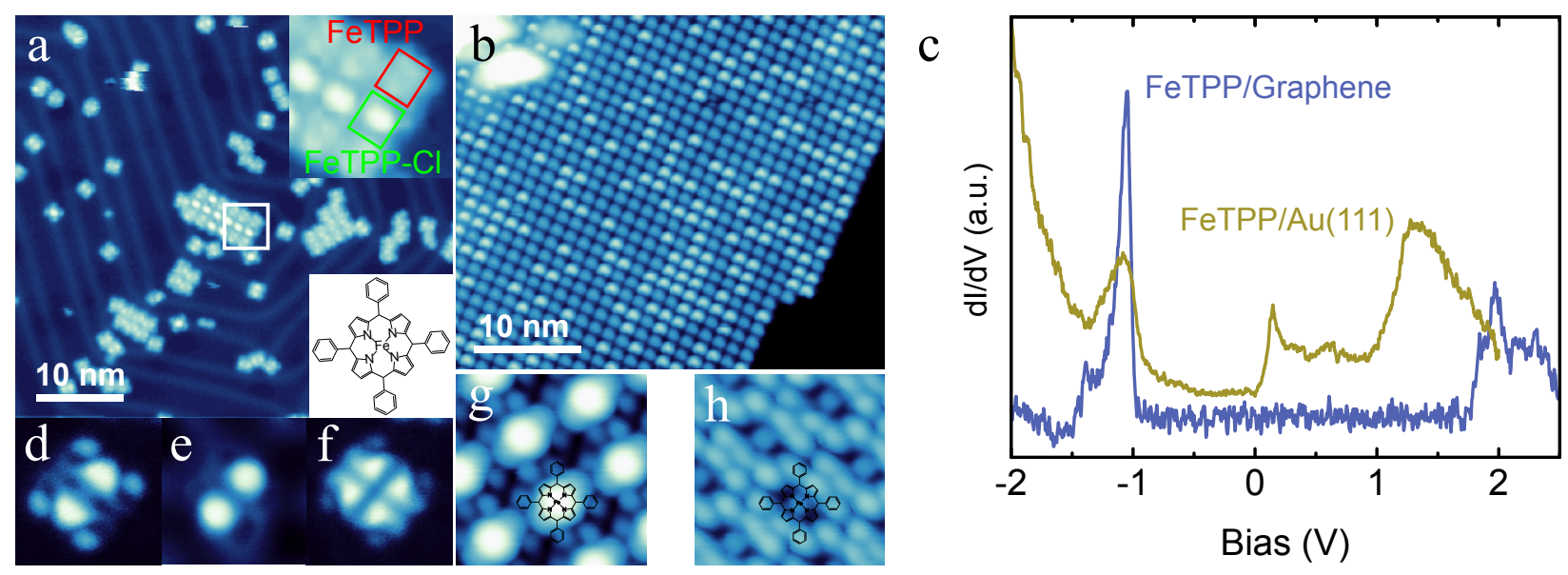

Figure 1: (a) STM image (1 V, $100 \mathrm{pA})$ of FeTPP molecules on $\mathrm{Au}(111)$. The inset on top is a zoomed image corresponding to the area marked by a square. The bottom inset is a scheme of the FeTPP molecule with the same orientation as the molecule shown in (d-f). (b) STM image $(-2 \mathrm{~V}, 50 \mathrm{pA})$ of FeTPP molecules on pristine graphene. (c) $\mathrm{d} I / \mathrm{d} V$ spectra of FeTPP on $\mathrm{Au}(111)$ and on pristine graphene showing a gap of $1.25 \mathrm{~V}$ on $\mathrm{Au}(111)$ and $2.9 \mathrm{~V}$ on graphene. (d-f) Conductance maps of a single FeTPP molecule on $\mathrm{Au}(111)$ measured at (d) $-1.1 \mathrm{~V}$, (e) $0.15 \mathrm{~V}$ and (f) $1.3 \mathrm{~V}$. (image size $3 \mathrm{~nm} \times 3 \mathrm{~nm}$ ). (g-h) STM image of FeTPP molecules on graphene at $(\mathrm{g})-1.1 \mathrm{~V}$ and $(\mathrm{h}) 2 \mathrm{~V}$. (image size $3.5 \mathrm{~nm} \times 3.5 \mathrm{~nm}$ ).

state as will be discussed below. At the center of the molecule, the peak at $0.15 \mathrm{~V}$ is not visible due to its localization on the pyrrole groups (see supporting information Figure S1). When the molecules are assembled in islands their spectroscopy is not noticeably modified as shown by $\mathrm{dI} / \mathrm{dV}$ measurements for molecules in islands (see supporting information Figure S2). When FeTPP molecules are adsorbed on pristine graphene, their electronic properties are very different. The HOMO-LUMO gap is larger on graphene as previously observed with other molecules. ${ }^{5,17}$ This is due to the gap renormalization for molecules on conducting substrates that reduces the gap due to image charge. On graphene the molecule-surface interaction is weaker, therefore the gap reduction is smaller, leading to a larger gap on graphene than on $\mathrm{Au}(111)$. More importantly, the topographs of FeTPP in Figure 1g and Figure $1 \mathrm{~h}$ at $-1.1 \mathrm{~V}$ and $2 \mathrm{~V}$ close to the HOMO and LUMO state on graphene (measured at $-1.05 \mathrm{~V}$ and $1.85 \mathrm{~V}$ ) show that the frontier orbitals do not have the same nature on graphene and on $\mathrm{Au}(111)$. Note that the topographical images of FeTPP on graphene at the energy of the HOMO and LUMO state is indicative of the shape of the molecular states, since the 
total current at these sample bias is dominated by the amplitude of the $\mathrm{d} I / \mathrm{d} V$ signal of the HOMO or LUMO state (the $\mathrm{d} I / \mathrm{d} V$ signal in the gap is nearly zero). The most striking difference between the molecule on $\mathrm{Au}(111)$ and on graphene, is that the HOMO is localized on the iron atom when the molecule is on graphene. This state is totally absent on $\mathrm{Au}(111)$ where all the molecular states are localized on the macrocycle. The LUMO, however, is also located on the macrocycle when the molecule is on graphene similarly to the $\mathrm{Au}(111)$ case. The origin of these features will be explained in the following.
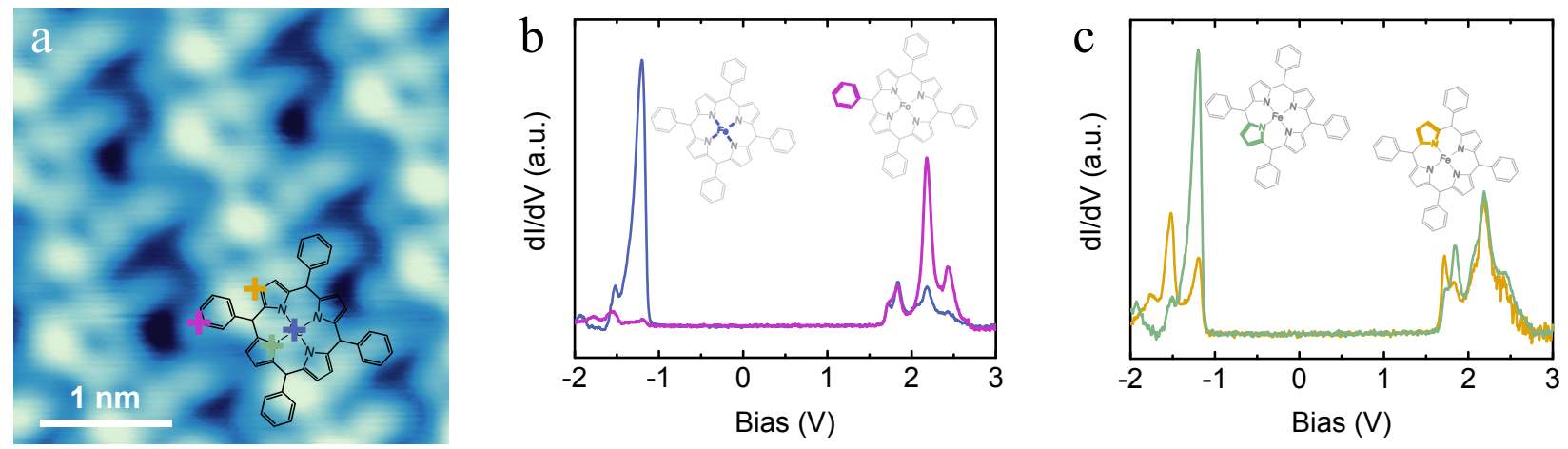

Figure 2: (a) STM image (1.9 V, 5 pA) of self-assembled FeTPP molecules on pristine graphene. The scheme of the molecule is a guide to the eye for the molecule orientation. (b-c) $\mathrm{d} I / \mathrm{d} V$ spectra measured at different positions of a FeTPP molecule. The positions are indicated by colored marks in (a) which correspond to colored groups in the molecular schemes as shown in the inset of (b) and (c).

A closer look at the spectroscopy of FeTPP on graphene (Figure 2) reveals another essential difference between the molecules on graphene and $\mathrm{Au}(111)$. While the spectroscopy on $\mathrm{Au}(111)$ shows distinct broad peaks, the spectrum on graphene exhibits a fine structure made of a series of sharp peaks in close proximity to the HOMO and LUMO states. Such peaks may originate from vibronic transitions, ${ }^{18-20}$ however we rule out this possibility because the peaks which are close in energy show different spatial localization as will be shown below. The spectrum on graphene can therefore be described by the HOMO-1 (-1.5 V), HOMO (-1.2 V), LUMO (1.7 V), LUMO+1 (1.8 V) and LUMO+2 (2.2 V) (Figure 2). The dependence of the peaks intensity on the tip location reveals that the states at $1.7 \mathrm{~V}$ and 1.8 $\mathrm{V}$ are localized on pyrrole groups while the states at $2.2 \mathrm{~V}$ is localized on the phenyl groups. 
The HOMO state at $-1.2 \mathrm{~V}$ is clearly located on the Fe atom, as seen in Figure $2 \mathrm{~b}$, while the HOMO-1 state appears on opposite pyrrole groups as seen in Figure 2c. To see more clearly the localization of the states, $\mathrm{d} I / \mathrm{d} V$ maps were measured. For better observation of the state at the single molecule level, we performed measurements on nitrogen doped graphene where the spectroscopy of molecules above a nitrogen atom undergoes a downshift. This shift is due to a fractional charge transfer between graphene and molecules at nitrogen sites, that has been observed for several molecules on doped graphene. ${ }^{2,5,20}$ Here, this effect allows us to visualize the states of one molecule in the molecular lattice and to avoid to mix these with the states of its neighbors. ${ }^{5}$ Indeed, on pristine graphene, the visualization of resonant state of a molecule is difficult because all molecules have a resonance at the same energy. Therefore, a dI/dV map will mix the image of the state of the investigated molecule with the image of state of the neighboring molecules. For a molecule which is above a nitrogen atom the energies of the molecular states are shifted. As a consequence, an image at the energy of a molecular state of the molecule above nitrogen reveal only the state of that molecule without contribution of the surrounding molecules.

In Figure 3a we show a molecular island on nitrogen doped graphene. A sequence of imaging, spectroscopy and removal of molecules with the STM tip allows to locate the nitrogen atoms below the molecules that are marked by red dots in Figure 3a (see also Supporting Information Figure S3). The $\mathrm{d} I / \mathrm{d} V$ spectra measured for molecules adsorbed above a nitrogen and a carbon site clearly reveals a downshift of the molecular spectrum on nitrogen (Figure $3 \mathrm{~b}$ ). We show in Figure $3 \mathrm{c}$ the $\mathrm{d} I / \mathrm{d} V$ spectra and maps, that confirm the localization of the states described above. The HOMO state on graphene is clearly localized on the Fe atom, while this state is not observed on $\mathrm{Au}(111)$. The HOMO-1 on graphene is localized on two opposite pyrroles. Note that measurements on pristine graphene show the same localization of the molecular states despite a loss of clarity due to a mixing of images of neighboring molecules (see Supporting Information Figure S4) . On $\mathrm{Au}(111)$ it is the HOMO state that shows such a localization. These features reveal that the molecular 

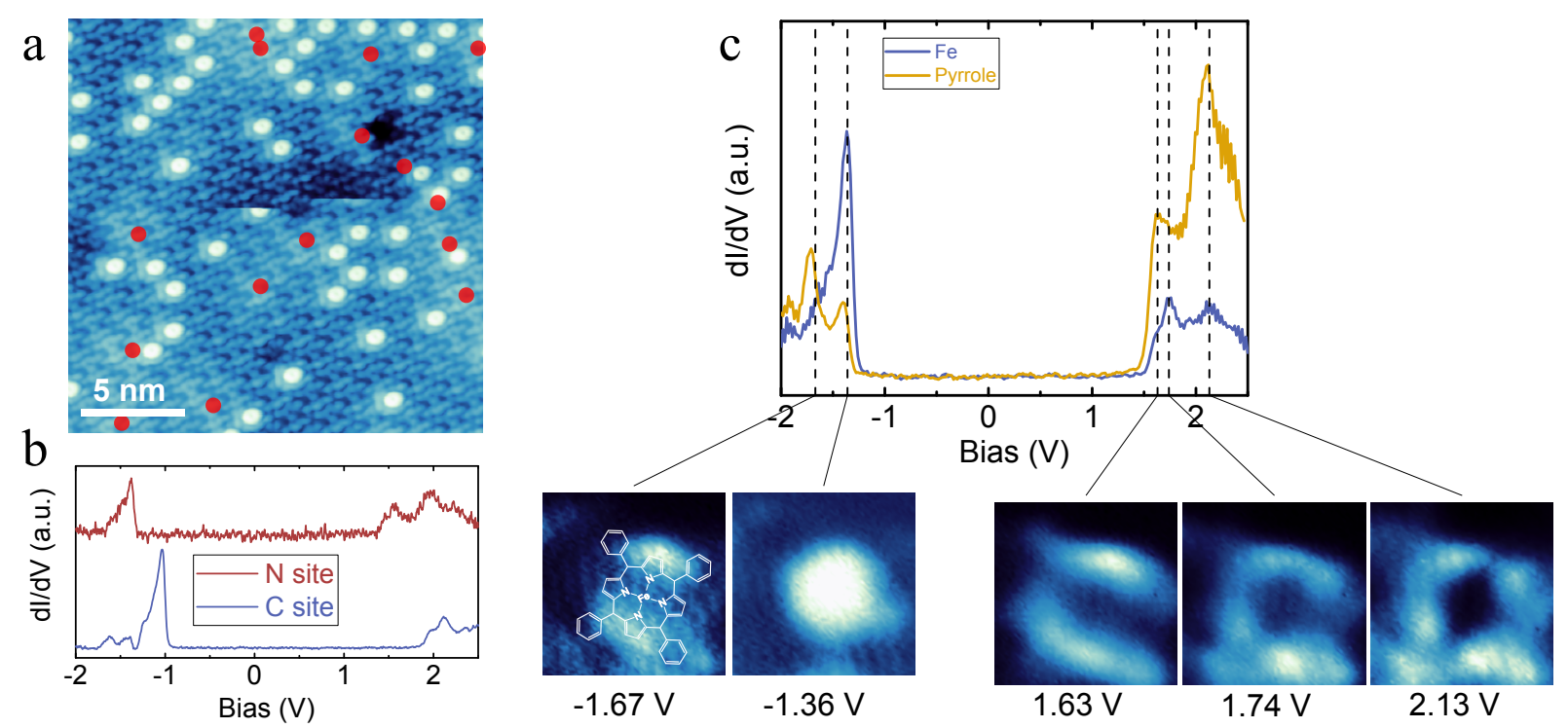

Figure 3: (a) STM image (1.9 V, 5 pA) of a FeTPP island on nitrogen-doped graphene. Red dots indicate the position of nitrogen atoms below the molecular islands. (b) $\mathrm{d} I / \mathrm{d} V$ spectra of a FeTPP molecule above a nitrogen site (red curve) and a carbon site (blue curve). (c) $\mathrm{d} I / \mathrm{d} V$ spectra of a FeTPP molecule adsorbed on a nitrogen site measured above the Fe atom and above a pyrrole group. The bottom part shows the $\mathrm{d} I / \mathrm{d} V$ maps at bias voltages corresponding to the peaks observed in the $\mathrm{d} I / \mathrm{d} V$ spectra (shown above) and marked by vertical dashed lines in the graph (image size $1.8 \mathrm{~nm} \times 1.8 \mathrm{~nm}$ ). 
states of physisorbed FeTPP emerge from different molecular orbitals when the molecule is on $\mathrm{Au}(111)$ or graphene.

To interpret our experimental results we have carried out DFT calculations for both free and deposited FeTPP molecules (see Methods for computational details). We have applied a Hubbard-like correction of $\mathrm{U}=5 \mathrm{eV}$ to Fe d orbitals which is close to the one used previously in Ref.[ 16]. This value seems to be reasonable (we have also tried to calculate U and have found values in the range between 5 and $5.5 \mathrm{eV}$ ) and will reproduce at the same time correct placement of Fe-related HOMO orbitals and energetics of molecular states as we will see in the following. Our calculations showed that the high spin solutions with $S=2$ are systematically higher in energy compared to those of $S=1$ (of about $0.16 \mathrm{eV}$ for FeTPP on $\mathrm{Au}(111)$ ) (see Supporting Information Figure S5), therefore we will focus on $S=1$ states only in the following. As was already mentioned above, for a free FeTPP molecule many realizations of $S=1$ state are possible according to different occupations of Fe $d$ orbitals (a more exhaustive study of the different occupations is also presented in the Supporting Information Table S1) as schematically shown in Figure 4a. Our DFT calculations confirm this picture. We find that the $d_{z^{2}}$ and degenerate (by symmetry) $d_{x z, y z}$-originated orbitals are very close in energy. Therefore, the three electronic configurations are possible where the spin down electron is placed on one of those orbitals. The state 1 (with doubly occupied $d_{z^{2}}$ orbital) was found to have the lowest energy which is in consistent with previous reported calculations. ${ }^{12,13}$ It is worth noticing that, since $d_{x z^{-}}$and $d_{y z}$-originated orbitals are degenerate, another state can be realized where a spin down electron occupies an orbital which is a linear combination (with the same weight) of the two above orbitals (state 4). We find that this symmetric realization is energetically favorable and results in fact in the lowest energy state, as summarized in Figure 4c. However, this state cannot be realized on a substrate, as will be discussed below.

When the molecule is deposited on a substrate it is deformed and adopts a saddle-like 
a)

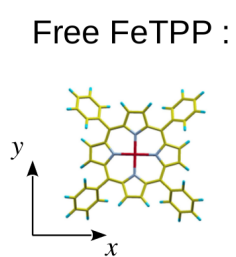

b)

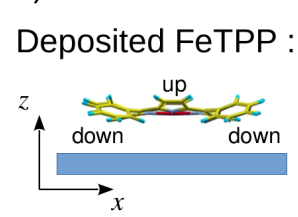

c)
State \#1
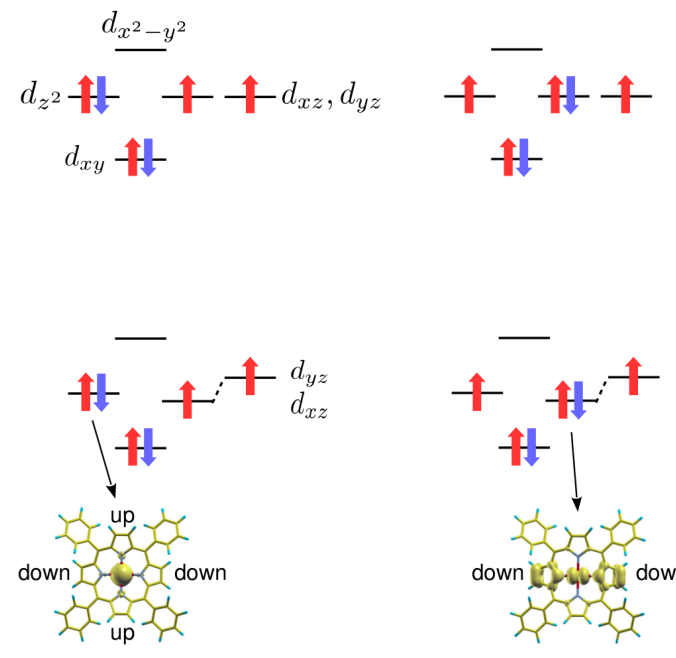
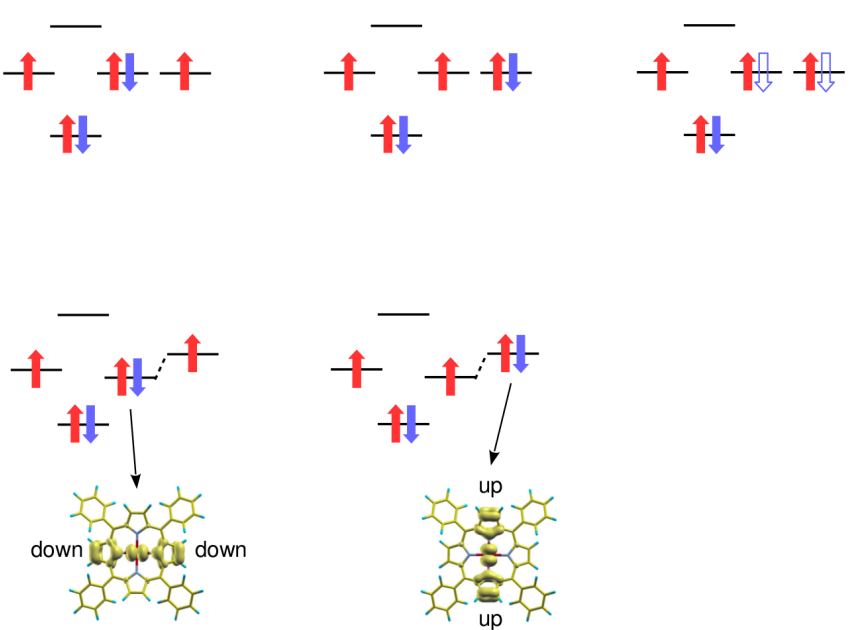

\#3

\#4

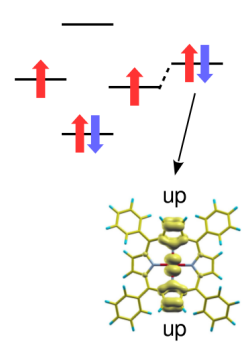

\begin{tabular}{l|lllc} 
& State 1 & State 2 & State 3 & State 4 \\
\hline free FeTPP & 0.0 & 53.6 & 53.6 & -28.8 \\
FeTPP/Gr & $0.0(0.0)$ & $4.5(64.2)$ & $21.7(75.1)$ & -- \\
FeTPP/Au & $0.0(0.0)$ & $-3.9(76.8)$ & $41.2(68.1)$ & --
\end{tabular}

Figure 4: Schematic representation of various $\mathrm{S}=1$ electronic states of (a) isolated and (b) deposited FeTPP molecule; (c) total energies (in meV) of different solutions (the energy of the state 1 is set to 0 ) for isolated and deposited molecules. The total energies of molecular subsystem (with removed substrate) are also given in parenthesis. 
shape with two pyrroles pointing upwards and two downwards to the substrate (Figure 4b). ${ }^{21}$ Its symmetry is therefore reduced so that only two mirror planes, $X Z$ and $Y Z$ (perpendicular to the molecule), are left. This leads to the splitting of $d_{x z, y z}$-originated orbitals. The state 2 with fully occupied $d_{x z}$ orbital (spread over downward pyrroles) gains much more energy from electronic hybridization with a substrate compared to both states 3 (with occupied $d_{y z}$ orbital localized on upward pyrroles) and 1 (with strongly localized on Fe $d_{z^{2}}$ orbital). In addition, a reduction of Coulomb energy due to more delocalized nature of $d_{x z}$ orbital (more penetrating into the substrate) also favors the state 2 over the state 1 . This overall gain of energy is larger for the FeTPP on Au, where Fe/substrate distance is $2.7 \AA$, than on graphene, where it is $3.1 \AA$. We find that for graphene state 2 is only $4.5 \mathrm{meV}$ higher in energy than state 1 and is lower (by $3.9 \mathrm{meV}$ ) for $\mathrm{Au}$ (Figure 4c). Concerning the symmetric state 4, discussed for the free molecule, we argue that it could be hardly realized for deposited FeTPP molecules because of the pronounced splitting of the $d_{x z, y z}$ orbitals and reduced symmetry of the system (we find, in particular, that for FeTPP/Au case it does not converge during the self-consistent DFT run). It should be noted that an accurate description of the closely placed states 1 and 2 may be quite delicate within standard DFT and may require a more sophisticated treatment with more advanced and computationally demanding functionals (like hybrid, vdW-DFT, or others). We find, in particular, that their relative energy positions depend on both the choice of value for the Hubbard $U$ and the molecule/substrate distance: the larger $U$ favors state 1 while the shorter distance favors state 2 (see Supporting Information Figure S6). Therefore, our DFT results should be taken with a care and considered rather as providing a general trend.

Based on the above analysis, we suggest that the FeTPP molecule preserves its freestanding electronic configuration (state 1) on graphene but switches to the state 2 when deposited on $\mathrm{Au}(111)$ due to stronger electronic interactions with the substrate. We present in Figure 5 the corresponding molecular DOS as well as DOS projected onto Fe d-orbitals. For the molecule on $\mathrm{Au}$, the $d_{x z}$-originated spin down orbital, being localized on downward 


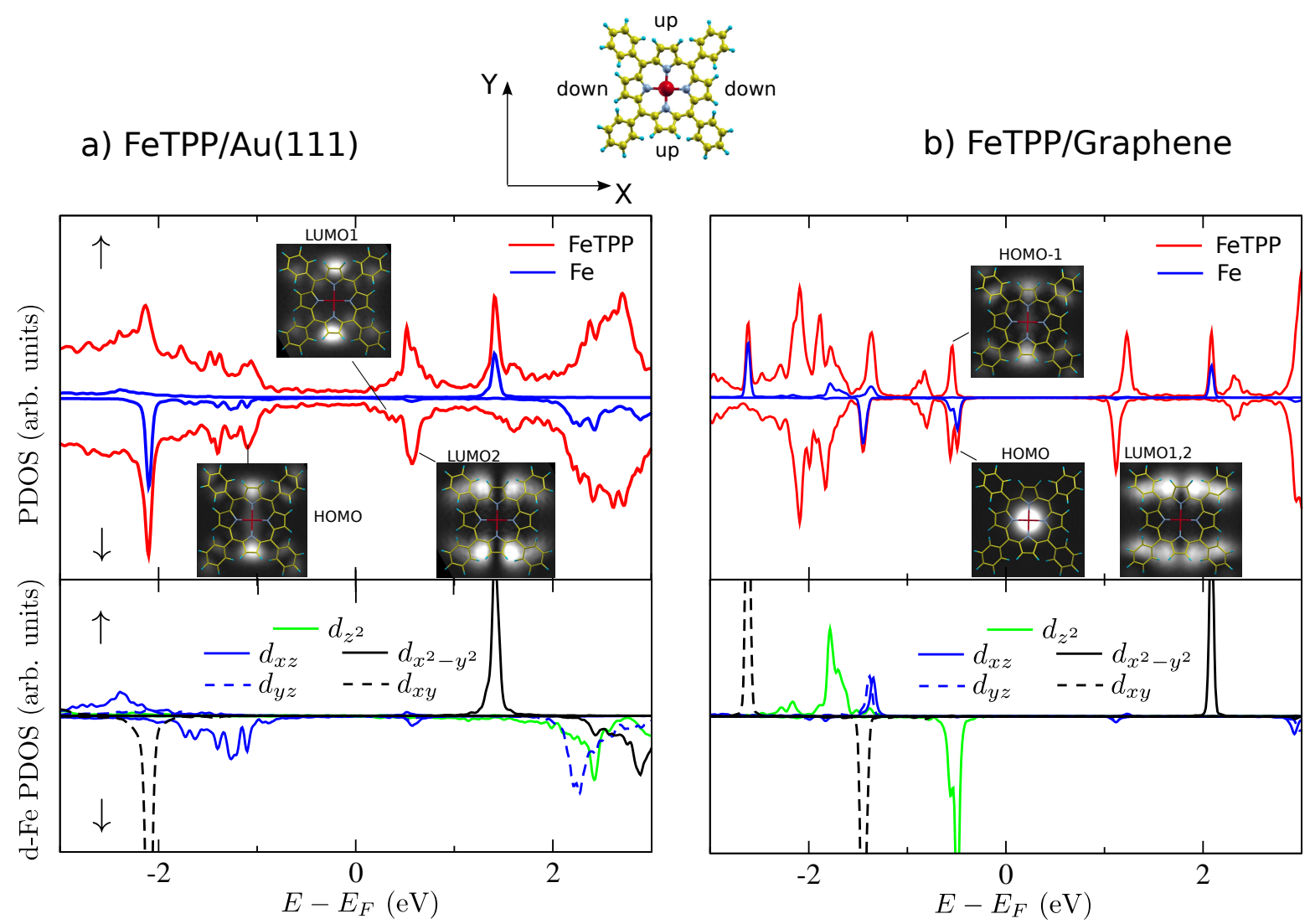

Figure 5: Calculated molecular and Fe DOS as well as the DOS projected onto different Fe d-orbitals (PDOS) for (a) FeTPP/Au in state 2 and (b) FeTPP/Graphene in state 1 (b). Spin up and down components are shown with positive and negative sign, respectively. Calculated from vacuum DOS (at $4 \AA$ above the molecule) STM images are also presented for selected energies. 
pyrols, as expected hybridizes strongly with the substrate states. It appears as a rather broad feature in the spin down $d_{x z}$ PDOS at around $-1.2 \mathrm{eV}$. The simulated STM image at this energy (calculated from the vacuum LDOS $4 \AA$ above the molecule) is therefore dominated by (unpolarized) pyrrole states appearing at the same energy and showing up as two bright lobes in the STM image (see Supporting Information Figure S7 for the molecular orbitals of free FeTPP molecule). For graphene, molecular states are much less coupled to the substrate. The well-defined spin down HOMO orbital originates from the Fe $d_{z 2}$ orbital. Its simulated STM image clearly shows the circular shape centered above the Fe atom and characteristic to the $d_{z^{2}}$ orbital. As for $\mathrm{Au}$, right below the HOMO we recover non-Fe molecular states showing the same two lobes structure. Altogether, our theoretical results are thus in a rather good agreement with experimental observations. We find, moreover, that the FeTPP LUMO orbital appears very close to the Fermi level in the case of Au. Careful analysis (see Supporting Information Figure S7) reveals that it consists of two degenerate levels, LUMO1 and LUMO2, which become split for state 2 (on $\mathrm{Au}$ ) but not for state 1 (graphene). In case of Au, LUMO1 is shifted closer to the Fermi level, it strongly hybridizes with the Au surface appearing therefore as a broad feature in molecular DOS. It shows two lobes shape on upward pyrroles. On the contrary, LUMO2 hybridizes less with the substrate and therefore produces a more pronounced peak in molecular DOS. It shows a kind of antibonding shape on upward pyrroles. These shapes of two LUMO orbitals can be clearly seen in the simulated STM images calculated at specific energies as shown in Figure 5a. Note that very similar behaviour of two-fold degenerate LUMO upon adsoption was previously reported by us for $\mathrm{H}_{2} \mathrm{TPP}$ on $\mathrm{Au}(111)$ (see Fig.S4 of Ref. 21). We therefore suggest that the states probed by STM at 0.15 and $1.3 \mathrm{eV}$ above the Fermi energy are associated with the LUMO1 or LUMO2 molecular orbitals, respectively. It should be pointed out that the position of the LUMO orbitals is rather sensitive to the number of Au layers used in the calculation. Increasing the number of layers shifts them closer to the Fermi level in better agreement with the experiment (see Supporting Information Figure S8). In case of graphene, 
the two LUMO are almost degenerate (Figure 5b) and their collective shape agrees quite well with the STM image acquired at $1.9 \mathrm{~V}$ (Figure 2a).

We have investigated the electronic coupling of FeTPP molecules with $\mathrm{Au}(111)$ and graphene. The molecule-substrate interaction leads to different frontier orbitals depending on the substrate: on $\mathrm{Au}(111)$ the HOMO state is localized on the organic core, while on graphene it is localized on the iron atom and mainly involves its $d_{z^{2}}$ state. The origin of this difference derives from the interplay between the spatial localization of Fe-originated molecular orbitals and their coupling strengths to the substrate determining the final electronic configuration. This shows how the molecule-substrate coupling can tune the frontier orbitals opening a promising route toward interfacial engineering in molecular electronics.

\section{Acknowledgement}

This project received funding from the European Unions Horizon 2020 research and innovation programme under Grant Agreement No. 766726. The Center for Nanostructured Graphene (CNG) is sponsored by the Danish Research Foundation (DNRF103).

\section{Methods}

Multilayer $(\approx 5-10)$ graphene samples were obtained on $\operatorname{SiC}(000 \overline{\mathbf{1}})$ by annealing the substrates in ultrahigh vacuum (UHV) at $1320^{\circ} \mathrm{C}$ for 12 min under a silicon flux of $\approx 1 \mathrm{ML} / \mathrm{min}$. The nitrogen-doping was performed by exposing the graphene sample to a flux of nitrogen radicals produced by a remote radio-frequency plasma source. ${ }^{22}$ Pristine and nitrogen-doped graphene samples were then transferred in air to a UHV chamber and degassed at $\sim 800^{\circ} \mathrm{C}$ for a few minutes before the measurements. FeTPP (purity >94\%) molecules (Aldrich) were sublimated using an effusion cell (Dr. Eberl MBE-Komponenten GmbH) under vacuum at $275^{\circ} \mathrm{C}$ onto the samples at the STM stage maintained at $\approx 5 \mathrm{~K}$. The graphene samples were then brought to room temperature allowing molecules to self-assemble and form a 2D 
island on the surface. All STM measurements were performed with a low-temperature STM apparatus (Omicron) working at $4.6 \mathrm{~K}$ at a pressure lower than $1 \times 10^{-10} \mathrm{mbar}$. The $\mathrm{d} I / \mathrm{d} V$ spectra were acquired using a lock-in detector at a frequency of ca. $823 \mathrm{~Hz}$ and a modulation amplitude of $35 \mathrm{mV}$. The measurements were performed with an electrochemically etched tungsten tip. Before measuring on graphene, the tip was calibrated on a $\mathrm{Au}(111)$ surface until it showed the Shockley surface state feature in the spectroscopic measurements.

The DFT calculations were carried out using plane wave electronic structure package Quantum ESPRESSO (QE). Local density approximation (LDA) in the Perdew-Zunger parametrization (PZ) for exchange-correlation functionals. The FeTPP/Au system was simulated by three $\mathrm{Au}(111)$ layers with the molecule adsorbed on one side and (8x8) in-plane supercell was employed in order to avoid artificial molecule-molecule interactions. For FeTPP on Graphene (10x10) unit cell was used. The total energy calculation of various $\mathrm{S}=1$ solutions was implemented in two steps: i) a self-consistent run is performed with imposed occupations of Fe d-orbitals using a penalization technique (by adding terms like $\lambda\left(n_{i}-n_{i 0}\right)^{2}$ to the total energy functional where $n_{i}$ and $n_{i 0}$ are actual and desired occupations of i-th d-orbital, respectively, and $\lambda$ is a penalty coefficient); ii) the converged charge density is read and used as the starting guess for another self-consistent run without any constraint producing the final total energy of a state with desired occupations. For each electronic configuration atomic relaxations were performed, two Au layers were kept fixed while the molecule and the surface layer in contact with it were allowed to relax until atomic forces became lower than $10^{3} \mathrm{Ry} /$ bohr. For Graphene all the atoms were allowed to relax. We also took partially into account Van der Waals interactions using semi-empirical dispersion corrections (DFT-D) as was formulated by Grimme ${ }^{23}$ and is implemented in QE package. All geometry optimizations were performed using only 1 (shifted) k-point while for electronic structure analysis more dense (6x6) k-point grid was employed and five layers of $\mathrm{Au}$ were used. The plane wave cut-off energies of 30 and 300 Ry were used for electronic wave-functions and charge 
density, respectively.

\section{Supporting Information Available}

Spectroscopy of FeTPP on $\mathrm{Au}(111)$, Localization of nitrogen atoms below a molecular layer, Conductance maps on pristine graphene, Structural properties of FeTPP on Au(111) and Graphene, Interplay between two $\mathrm{S}=1$ solutions as a function of molecule/substrate distance and Hubbard U, Electronic structure of free-standing FeTPP in two $\mathrm{S}=1$ states, complementary QuantumATK calculations

\section{References}

(1) de la Torre, B.; Švec, M.; Hapala, P.; Redondo, J.; Krejčí, O.; Lo, R.; Manna, D.; Sarmah, A.; Nachtigallová, D.; Tuček, J. et al. Non-covalent control of spin-state in metal-organic complex by positioning on N-doped graphene. Nat. Commun. 2018, 9, $1-9$.

(2) Pham, V. D.; Ghosh, S.; Joucken, F.; Pelaez-Fernandez, M.; Repain, V.; Chacon, C.; Bellec, A.; Girard, Y.; Sporken, R.; Rousset, S. et al. Selective control of molecule charge state on graphene using tip-induced electric field and nitrogen doping. NPJ $2 D$ Mater. Appl. 2019, 3, 1-5.

(3) Sau, J. D.; Neaton, J.; Choi, H. J.; Louie, S. G.; Cohen, M. L. Electronic energy levels of weakly coupled nanostructures: C 60-metal interfaces. Phys. Rev. Lett. 2008, 101, 026804 .

(4) Barth, J. V. Fresh perspectives for surface coordination chemistry. Surf. Sci. 2009, 603, $1533-1541$. 
(5) Pham, V. D.; Lagoute, J.; Mouhoub, O.; Joucken, F.; Repain, V.; Chacon, C.; Bellec, A.; Girard, Y.; Rousset, S. Electronic interaction between nitrogen-doped graphene and porphyrin molecules. ACS Nano 2014, 8, 9403-9409.

(6) Tsiper, E.; Soos, Z.; Gao, W.; Kahn, A. Electronic polarization at surfaces and thin films of organic molecular crystals: PTCDA. Chem. Phys. Lett. 2002, 360, 47-52.

(7) Martnez-Galera, A. J.; Nicoara, N.; Martnez, J. I.; Dappe, Y. J.; Ortega, J.; GmezRodrguez, J. M. Imaging Molecular Orbitals of PTCDA on Graphene on Pt(111): Electronic Structure by STM and First-Principles Calculations. J. Phys. Chem. C 2014, $118,12782-12788$.

(8) Auwärter, W.; Écija, D.; Klappenberger, F.; Barth, J. V. Porphyrins at interfaces. Nat. Chem. 2015, 7, 105-120.

(9) Walker, F. A.; Simonis, U. Encyclopedia of Inorganic and Bioinorganic Chemistry; John Wiley \& Sons, Ltd: Chichester, UK, 2011.

(10) Collman, J. P.; Hoard, J.; Kim, N.; Lang, G.; Reed, C. A. Synthesis, stereochemistry, and structure-related properties of. alpha.,. beta.,. gamma.,. delta.tetraphenylporphinatoiron (II). J. Am. Chem. Soc. 1975, 97, 2676-2681.

(11) Boyd, P. D.; Buckingham, D. A.; McMeeking, R. F.; Mitra, S. Paramagnetic anisotropy, average magnetic susceptibility, and electronic structure of intermediate-spin $S=1(5$, 10, 15, 20-tetraphenylporphyrin) iron (II). Inorg. Chem. 1979, 18, 3585-3591.

(12) Liao, M.-S.; Scheiner, S. Electronic structure and bonding in metal porphyrins, metal= Fe, Co, Ni, Cu, Zn. J. Chem. Phys. 2002, 117, 205-219.

(13) Liao, M.-S.; Watts, J. D.; Huang, M.-J. Electronic structure of some substituted iron (II) porphyrins. Are they intermediate or high spin? J. Phys. Chem. A 2007, 111, $5927-5935$. 
(14) Gopakumar, T. G.; Tang, H.; Morillo, J.; Berndt, R. Transfer of Cl ligands between adsorbed iron tetraphenylporphyrin molecules. J. Am. Chem. Soc. 2012, 134, 1184411847.

(15) Rubio-Verdú, C.; Sarasola, A.; Choi, D.-J.; Majzik, Z.; Ebeling, R.; Calvo, M. R.; Ugeda, M. M.; Garcia-Lekue, A.; Sánchez-Portal, D.; Pascual, J. I. Orbital-selective spin excitation of a magnetic porphyrin. Commun. Phys. 2018, 1, 1-7.

(16) Wang, W.; Pang, R.; Kuang, G.; Shi, X.; Shang, X.; Liu, P. N.; Lin, N. Intramolecularly resolved Kondo resonance of high-spin Fe (II)-porphyrin adsorbed on Au (111). Phys. Rev. B 2015, 91, 045440 .

(17) Cho, J.; Smerdon, J.; Gao, L.; Suzer, O.; Guest, J. R.; Guisinger, N. P. Structural and electronic decoupling of $\mathrm{C} 60$ from epitaxial graphene on SiC. Nano Lett. 2012, 12, 3018-3024.

(18) Krane, N.; Lotze, C.; Reecht, G.; Zhang, L.; Briseno, A. L.; Franke, K. J. HighResolution Vibronic Spectra of Molecules on Molybdenum Disulfide Allow for Rotamer Identification. ACS nano 2018, 12, 11698-11703.

(19) Mehler, A.; Nel, N.; Krger, J. Dissimilar Decoupling Behavior of Two-Dimensional Materials on Metal Surfaces. J. Phys. Chem. Lett. 2020, 11, 5204-5211.

(20) Bouatou, M.; Mondal, S.; Chacon, C.; Joucken, F.; Girard, Y.; Repain, V.; Bellec, A.; Rousset, S.; Narasimhan, S.; Sporken, R. et al. Direct observation of the reduction of a molecule on nitrogen pairs in doped graphene. Nano Letters 2020, 20, 6908-6913.

(21) Pham, V. D.; Repain, V.; Chacon, C.; Bellec, A.; Girard, Y.; Rousset, S.; Smogunov, A.; Dappe, Y. J.; Lagoute, J. Control of molecule-metal interaction by hydrogen manipulation in an organic molecule. J. Phys. Chem. Lett. 2016, 7, 1416-1421. 
(22) Joucken, F.; Tison, Y.; Lagoute, J.; Dumont, J.; Cabosart, D.; Zheng, B.; Repain, V.; Chacon, C.; Girard, Y.; Botello-Méndez, A. R. et al. Localized state and charge transfer in nitrogen-doped graphene. Phys. Rev. B 2012, 85, 161408.

(23) Grimme, S. Semiempirical GGA-type density functional constructed with a long-range dispersion correction. J. Comput. Chem. 2006, 27, 1787-1799. 